\title{
Morfología y Repercusiones Diagnóstico-Terapéuticas de las Lesiones Preneoplásicas Gástricas
}

\author{
Morphology and Diagnostic Determinants of Gastric Precancerous Conditions
}

\author{
Sergio Sotelo ${ }^{1,2,3} \&$ Carlos Manterola ${ }^{1,4}$
}

\begin{abstract}
SOTELO, S. \& MANTEROLA, C. Morfología y repercusiones diagnóstico-terapéuticas de las lesiones preneoplásicas gástricas. Int. J. Morphol., 37(3):917-927, 2019.
\end{abstract}

RESUMEN: El carcinoma gástrico (CG) de tipo intestinal se origina en un epitelio displásico, que a su vez se desarrolla en medio de una atrofia gástrica (AG) y metaplasia intestinal (MI). La infección por Helicobacter pylori (HP) es la causa más frecuente de AG, causando una pangastritis atrófica multifocal. Entre otras condiciones que producen inflamación crónica de la mucosa gástrica se encuentran también la gastritis autoinmune y la anemia perniciosa. El marco conceptual sobre el cual descansa gran parte de la investigación actual y nuestra comprensión de los cambios que ocurren en la mucosa gástrica se debe a la denominada "cascada de Correa"; quien planteó que la mucosa gástrica crónicamente inflamada, da paso a la AG, que va adquiriendo focos de MI y en dicho epitelio se desarrollará finalmente una displasia (DIS). Se ha acuñado el término lesiones preneoplásicas gástricas (LPG), para referirse a: AG, MI y DIS.Después de la erradicación de HP, se ha demostrado una reducción general de la incidencia de CG; efecto que no es tan claro, cuando la pangastritis por HP ha evolucionado a AG extensa. De tal modo que el efecto de la erradicación de HP medido a través de EC, ha sido poco consistente. La AG grave diagnosticada por histología representa la condición de mayor riesgo. Por otra parte, la MI puede ser de tipo intestinal (delgado-entérica ó incompleta) y la colónica (colónica ó completa) considerándose a esta última, como la variedad de peor pronóstico. El diagnóstico histológico de este tipo de lesiones determina que quien las padece, debe someterse a vigilancia endoscópica. El objetivo de este manuscrito fue resumir la evidencia existente respecto de las LPG, en términos de su caracterización morfológica y sus repercusiones diagnóstico-terapéuticas (significado patológico, graduación del riesgo, vigilancia recomendada; y factores de riesgo).

PALABRAS CLAVE: Lesiones pre neoplásicas gástricas; Cáncer gástrico; Helicobacter pylori; Gastritis atrófica; Gastritis crónica activa no atrófica; Gastritis atrófica multifocal; "Metaplasia intestinal; Displasia gástrica.

\section{INTRODUCCIÓN}

En la práctica clínica, es frecuente recibir informes de endoscopías digestivas altas, como parte del estudio de los pacientes. La formación curricular de pregrado y posttítulo habitual, permite entender usualmente sin dificultades las conclusiones de dicho examen, pero no siempre se está familiarizado con la descripción y conclusiones del informe histológico anexo (Lage et al., 2016; Sugano, 2013).

Entre las lesiones que pueden ser informadas por el patólogo, merecen especial atención un grupo denominado "lesiones preneoplásicas gástricas" (LPG); que como su apelativo indica, son lesiones que potencialmente pueden progresar al desarrollo de un cáncer gástrico (CG) (Rollán et al., 2014).
Bajo el concepto de LPG, se agrupan las siguientes lesiones: Gastropatía atrófica (GA), metaplasia intestinal (MI) y displasia (DIS). A estas, se ha de sumar la infección de la mucosa gástrica por Helicobacter Pilorii (HP). El conocimiento de estas lesiones resulta crucial pues sólo de este modo se puede informar, educar y trazar un plan de vigilancia cuando se diagnostica alguna LPG (Sugano; Sung, 2016).

La primera descripción de una cascada de transformaciones de la mucosa gástrica desde la normalidad hasta el desarrollo de DIS, lo realizó Correa en 1975 (Correa et al., 1975). El mismo autor y Cols., realizaron las actualizaciones posteriores al modelo original (Correa, 1992, 1998;

\footnotetext{
${ }^{1}$ Programa de Magíster en Ciencias Médicas, Mención Cirugía, Universidad de La Frontera, Temuco, Chile.

${ }^{2}$ Facultad de Medicina, Universidad Católica del Maule, Talca, Chile.

${ }^{3}$ Hospital Regional de Talca, Talca, Chile.

${ }^{4}$ Centro de Excelencia en Estudios Morfológicos y Quirúrgicos (CEMyQ), Universidad de La Frontera, Temuco, Chile.
} 
Correa \& Piazuelo, 2012), agregando el rol del HP como principal causante de gastritis crónica activa; que conduciría a GA, la que a su vez llevaría al desarrollo de MI completa (MIC), MI incompleta (MII) y posteriormente a DIS. Evidencia posterior, ha dado sustento a esta teoría (AlAwadhi et al., 2011; Sampieri, 2013; Yakirevich \& Resnick, 2013; Abangah et al., 2016; Li et al., 2016).

Por otro lado asistimos a una creciente intención y necesidad de clasificar la intensidad de la existencia de dichas lesiones mediante la aplicación de un protocolo de toma de biopsias; cuyo objetivo es pronosticar el riesgo de desarrollar LPG en cada paciente en particular (Lage et al.).

El objetivo de este manuscrito fue resumir la evidencia existente respecto de las LPG, en términos de su caracterización morfológica y repercusiones diagnóstico-terapéuticas (significado patológico, graduación del riesgo, vigilancia recomendada; y factores de riesgo asociados).

\section{MATERIAL Y MÉTODO}

Diseño: Revisión narrativa.

Población: Se incluyeron revisiones sistemáticas (RS), ensayos clínicos (EC) y estudios observacionales; publicados entre enero de 2008 y diciembre de 2018; sin restricción idiomática; que incluyeran población humana que hubiese sido diagnosticada de LPG; que aportaren en los métodos de diagnóstico de las lesiones señaladas y de cómo optimizar la pesquisa de las mismas; relacionados con factores de riesgo de $\mathrm{CG}$, incluyendo condiciones como infección de la mucosa gástrica por HP; y ser familiar de primer grado de pacientes con CG. Se excluyeron artículos con aspectos moleculares de las LPG, por considerar que no aportan en práctica clínica.

Fuentes de información: Se revisaron los siguientes metabuscadores, bibliotecas y bases de datos: Trip Database, BIREME-BVS, SciELO, WoS, MEDLINE, EMBASE y SCOPUS.

Estrategias de búsqueda: En las fuentes antes señaladas, se realizaron búsquedas sensibles utilizando términos MeSH ("Precancerous Conditions"; "Stomach Neoplasms"; "Helicobacter pylori"; "Gastritis, Atrophic"; "Metaplasia"); palabras libres (Gastric precancerous conditions; Gastritis chronic active non atrophic; Gastritis atrophic multifocal; Intestinal metaplasia; Gastric dysplasia) y conectores booleanos (AND y OR); utilizando estrategias adaptadas a cada fuente de información.
Protocolo de estudio: La recuperación de documentos se cerró el 31 de diciembre de 2018. De los artículos seleccionados, se analizaron en orden progresivo títulos, resúmenes y artículo en extenso; en búsqueda del cumplimiento de los criterios de inclusión y exclusión.

Variables de interés: Gastritis crónica atrófica (gastritis atrófica), MI (completa e incompleta), DIS, e infección por HP.

\section{RESULTADOS}

Con las estrategias utilizadas en las fuentes de información, se logró recuperar 135 artículos, los cuales fueron analizados a título y resumen; lo que permitió seleccionar 53 artículos, que fueron revisados a texto completo siendo el insumo último del presente reporte. Con ellos, se sintetiza la evidencia respecto de las LPG y aspectos relacionados relevantes:

Gastritis crónica atrófica (Gastritis atrófica): Corresponde a la disminución o desaparición de las glándulas gástricas normales y su reemplazo por proliferación de tejido conectivo, infiltrado inflamatorio linfoide, o por estructuras glandulares diferentes a las normales del estómago, asociado a la expansión de la lámina propia (Fig. 1) (Rugge et el., 2008). La causa de la aparición de AG, precedida de la gastritis crónica activa, es principalmente la infección por HP que se sabe que infecta alrededor del $60 \%$ de la población mundial; explicando más del $95 \%$ de la etiología de la AG. Otra causa, de menor relevancia, es la gastritis de origen autoinmune con menos del $5 \%$ como etiología de la AG (Toh, 2014); de hecho, la prevalencia de esta en Europa (determinada por un estudio de cohortes multicéntrico), se ha establecido en 2,6\% utilizando como instrumento diagnóstico el protocolo Sidney (Wolf, 2014). Esta atrofia se expresa en hipoclorhidria e hipergastrinemia, como consecuencia de la disminución del capital glandular normal. La prevalencia de GA ha sido estimada a través de una RS en 33,4\% de la población general; estudio que aportó información respecto a que este valor puede cambiar según la incidencia de CG, siendo de $41,7 \%$ en paises de alta incidencia; y de 22,8\% en aquellos de moderada-baja incidencia (Marques-Silva et al., 2014).

La GA es considerada una condición precursora de CG, con tasas de progresión superiores a 1,0 \% por año en períodos de seguimiento (Whiting et al., 2002; Vannella et al., 2010). En una serie posterior, con un seguimiento promedio de 7,5 años (4 a 23 años), en 200 pacientes con AG, se verificaron tasas de incidencia anual persona/año de 0,25 $\%$ (IC $95 \%$ : 0,067; 0,63\%); y 0,43 \% (IC $95 \%: 0,17 ; 0,89$ $\%$ ) para CG y DIS respectivamente (Lahner et al., 2015). 

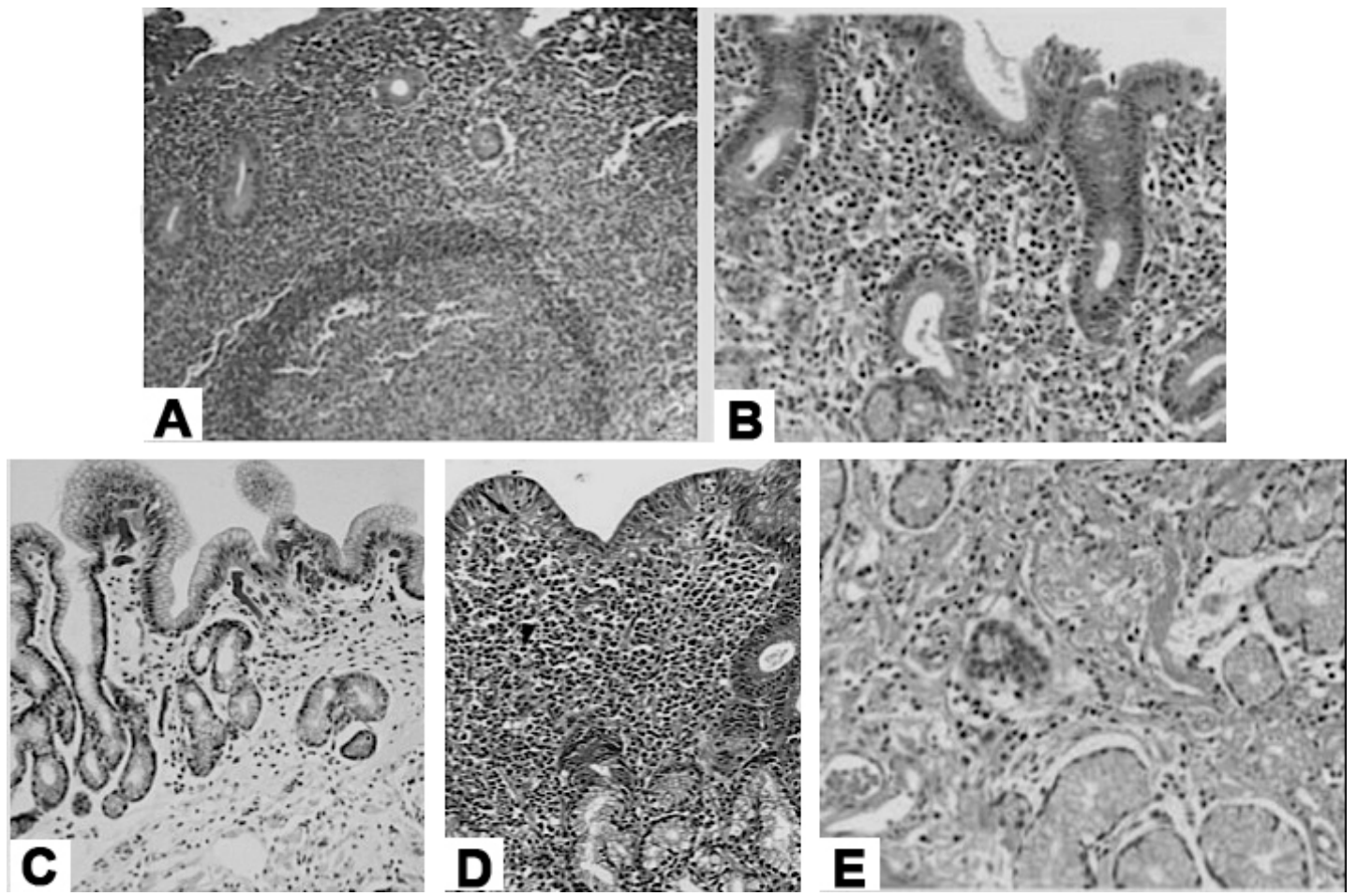

Fig. 1. Diferentes imágenes de aspectos morfológicos de gastritis crónica. A: Gastritis crónica (H\&E 20x); B: Gastritis crónica (H\&E 40x); C: Gastritis atrófica (pérdida multifocal de glándulas y reemplazo por fibrosis). D: Gastritis crónica activa no atrófica (infiltración del corion por linfocitos y macrófagos). E: Atrofia gástrica.

Metaplasia intestinal: Corresponde a la transformación de la mucosa gástrica, que sigue a la AG; como una lesión heterogénea según la histología y el tipo de mucina secretada con características que la acercan a la mucosa intestinal, de intestino delgado, de intestino grueso o mixta en caso de coexistir ambas (Fig. 2). Esta, puede ser completa (tipo I) e incompleta (tipo II). La MI se denomina como "limitada" cuando se encuentra circunscrita a una región del estómago; y como "extensa", en el caso de estar involucradas dos o más regiones del estómago. La prevalencia de MI en población general es de alrededor de $25,0 \%$; lo que varía según la incidencia de CG; de modo tal que en poblaciones con alta incidencia de $\mathrm{CG}$ es de $28,1 \%$ mientras que en poblaciones de incidencia moderada-baja de CG es de 21,7\% (Marques-Silva et al.). La MI histológicamente confirmada es el marcador más confiable de atrofia en la mucosa gástrica (Pimentel-Nunes et al., 2019).

a) MIC o tipo I: Se carecteriza por la pérdida de las glándulas gástricas y su reemplazo por estructuras glandulares con histoarquitectura de intestino delgado [células de absorción con márgenes en cepillo, células caliciformes y ocasionalmente Cellula panethensis (células de Paneth)]. Esa pérdida va acompañada de modificación de la función normal, por lo cual disminuye la secreción de ácido, con la consecuente hipoclorhidia. b) MII o tipo II: Se caracteriza por el reemplazo de epitelio por glándulas caliciformes con patrón arquitectónico semejante a la mucosa colónica con células mucosas híbridas con grandes vacuolas de diferentes tamaños, sin características de células absorbentes o células caliciformes.

En relación con el valor de diferenciar estos subtipos, con el objetivo de dar seguimiento más estricto a uno de ellos en pos de aumentar la pesquiza de CG; existe evidencia, a partir de una guía de práctica clínica, que sugiere que el valor de esto es limitado, poco consistente y además, presenta la dificultad de requerir técnicas de inmunohistoquímica para diferenciar los subtipos (DinisRibeiro et al., 2012). No obstante ello, en un estudio multicéntrico español, se observaron cifras que apoyan la diferenciación de subtipos, con un HR de progresión a CG de 2,75 (IC $95 \%$ 1,06; 6,26) para aquellos que al inicio del estudio eranportadores de MII respecto de aquellos con MIC con seguimiento medio de 12 años (González et al., 2016). Por otro lado, los resultados de una RS anterior, apoyan la utilidad de subtipificar MI como predictor de CG (González et al., 2013). En este mismo artículo, se plantea que la diferenciación de los subtipos de MI preceden históricamente al advenimiento de la inmunohistoquímica; siendo esta, una técnica requerida por los patólogos en la caracterización de la MI sólo en un bajo porcentaje de casos. Del mismo modo, 

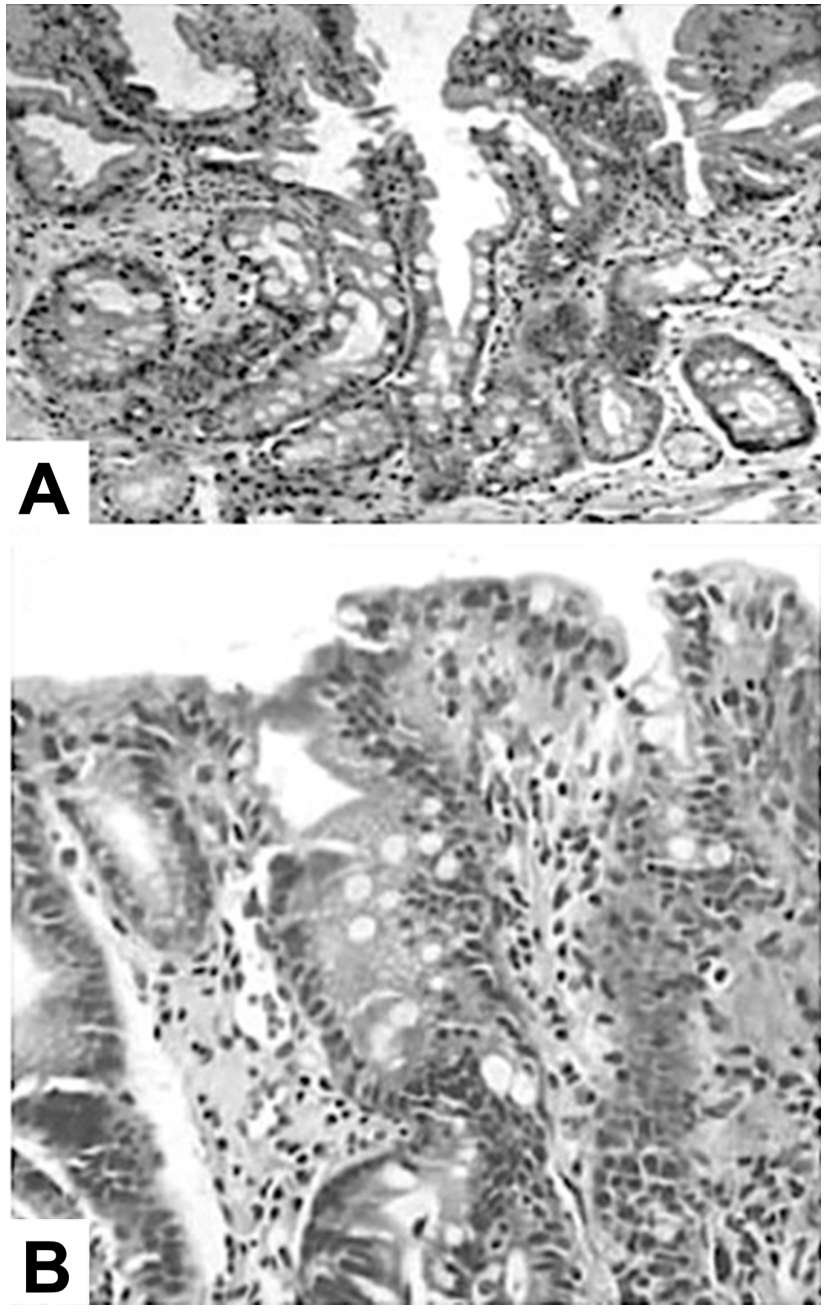

Fig. 2. Aspectos histológicos de metaplasia intestinal en mucosa gástrica. A: Metaplasia intestinal completa (H\&E 20x); B: Metaplasia intestinal completa (H\&E 40x).

determinaron que el riesgo de CG es de 4 a 11 veces mayor con MII en comparación con ausencia de MII (González et al., 2013). Asimismo, existe evidencia proveniente de un estudio de cohorte de pacientes con MI (no se aclara si eran tipo I o II), con seguimiento endoscópico e histopatológico seriado; con un promedio de 3,3 años tras el diagnóstico inicial de MI; que permitió objetivar que sólo un bajo porcentaje de pacientes $(2,7 \%)$, presentó progresión a DIS de bajo grado, DIS alto grado o carcinoma (den Hoed et al., 2013); sin embargo, se ha de considerar que este estudio fue conducido en una población de baja incidencia de CG.

Displasia: el término "displasia", derivado del griego y que significa "mala forma" o "mal modelo",; hace referencia a una anormalidad en el aspecto de las células debido a alteraciones en el proceso de maduración de las mismas. Se carecteriza por la combinación variable de alteraciones microscópicas confinadas a las glándulas, sin cruzar la membra- na basal a consecuencia de una maduración epitelial y proliferación celular anormal (Fig. 3). La DIS gástrica representa la penúltima etapa de la secuencia de carcinogénesis gástrica de acuerdo a la casacada de Correa. En un estudio de cohorte que dividió casos de DIS en 2 grupos (DIS leve-moderada y DIS grave), con seguimiento a 1,5 y 10 años se verificó CG en en el $2,1 \%$; $3,1 \%$; y $3,9 \%$ de pacientes que al inicio tenían DIS leve-moderada; y de $24,9 \% ; 29,5 \%$; y $32,7 \%$ de pacientes con DIS grave al inicio del seguimiento $(\mathrm{p}<0,001)$ (de Vries et al., 2008). En un estudio de cohorte ya mencionado se observó que el 94,0 \% de los casos de DIS de bajo grado, regresaron (den Hoed et al., 2013). Los datos anteriores dan cuenta de una gran diferencia pronóstica del grupo de DIS de leve-moderada en contraste con el de DIS grave. Hasta hace unos años existía acuerdo entre las opiniones de expertos y guías de práctica clínica, en que la DIS de bajo grado debía ser seguida con endoscopías y estudio histológico cada un año; en tanto la DIS de alto grado requería de resección local. Sin embargo, han surgido reportes que evidencian que hasta en un $24,0 \%$ de DIS de bajo grado sometidas a resección endoscópica, progresaron a DIS de alto grado o carcinoma (Lim et al., 2014); con la consiguiente recomendación de que DIS de bajo grado sea evaluada una vez pesquizada con endoscopia de alta resolución e incluso sometida diretamente a resección endoscópica. En tanto que el 52,7 \% de los sujetos con DIS de alto grado, progresaron a carcinoma (números que avalan la conducta actual de resección endoscópica para las DIS de alto grado). En este mismo estudio, se identificaron, por medio de un análisis multivariado, las siguientes variables como asociadas al cambio en diagnósticos de DIS de bajo a alto grado: diámetro de lesión igual o mayor a 1,8 cm (OR: 1,6; IC95\% de 1,309; 1,955), desnivel tenue (OR: 1,66; IC95\% de 1,108; 2,49); y tipo macroscópico deprimido (OR: 2,991; IC95\%: 1,$894 ; 4,723)$. Y las siguientes variables como asociadas al cambio en diagnósticos de DIS alto grado a carcinoma: tipo macroscópicamente plano (OR: 1,796; IC95\% de $0,860 ; 3,751)$ (Lim et al.).

Infección por HP: Es la causa más frecuente de enfermedad crónica de la mucosa gástrica; asociándose a gastritis activa, úlcera péptica, adenocarcinoma gástrico y linfoma tipo MALT gástrico. La mayoría de los individuos infectados por HP mantiene una "gastritis superficial" crónica activa, que en general cursa de forma asintomática (Fig. 4). Sólo un $10 \%$ de los infectados con HP verá avanzar su gastritis crónica activa a AG grave en el trancurso de los años, con una incidencia anual de 1,0\% a 3,0 \% (Kuipers et al., 1995; Kuipers, 1998).

Por otra parte, existe evidencia que la erradicación de HP disminuye la aparición del adenocarcinoma gástrico del tipo difuso de Lauren, cuando dicha erradicación es hecha en pacientes sin LPG (Wong et al., 2004). En esa misma 

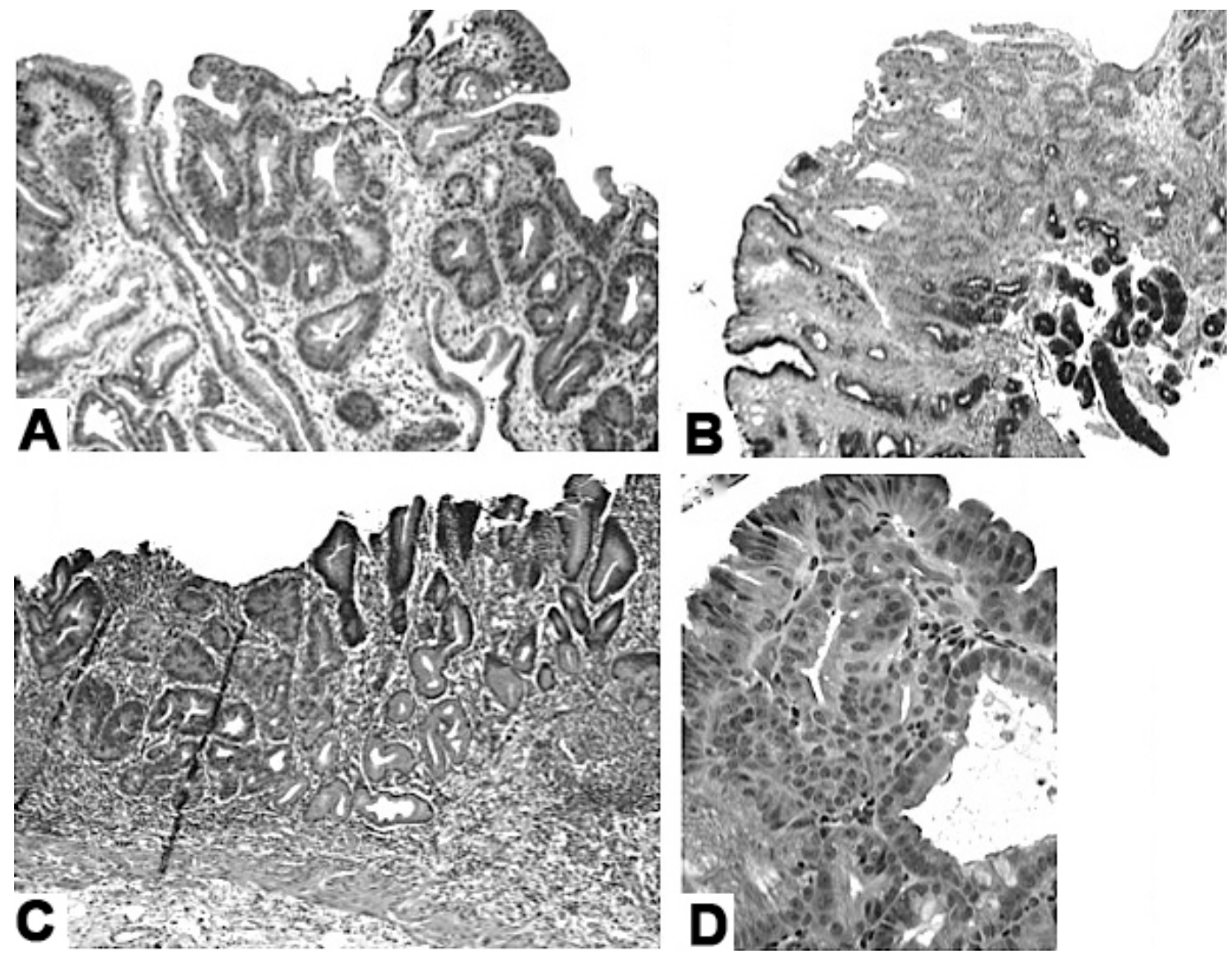

Fig. 3. Diversas imágenes de displasias gástricas. A: Displasia de bajo grado. B: Displasia de bajo grado, asociada a metaplasia intestinal (PAS para captar estructuras que secretan mucinas). C: Displasia de alto grado. D: Displasia de alto grado, con núcleos grandes e hipercromáticos; y nucléolos prominentes (H\&E 20x).

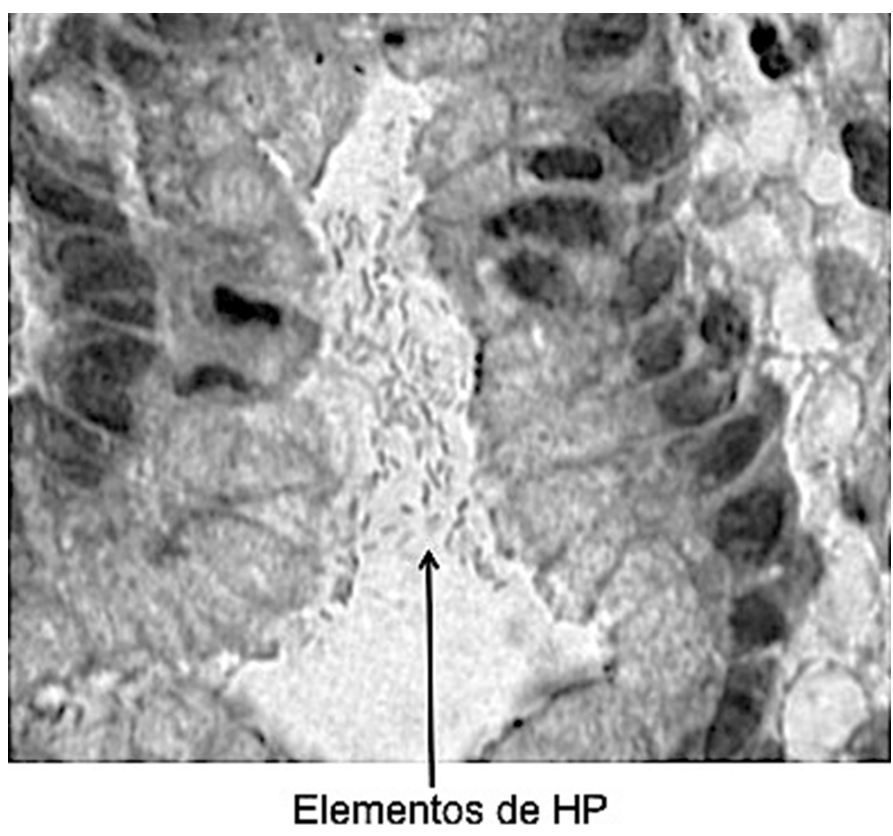

Fig. 4. Múltiples elementos bacterianos de HP (H\&E 200x). dirección, la erradicación de HP resultará más o menos eficiente para prevenir el desarrollo de CG en tanto se aplique en sujetos con menor grado de atrofia, lo que se verificó en mediciones realizada a nivel de fundus gástrico (Take et al., 2007; Yanaoka et al., 2009). Al respecto y de manera opuesta existen EC y RS con resultados que difieren a lo ya señalado (Fukase et al., 2008; Cho et al., 2013a). Las diferencias entre los estudios parecieran estar dados por inconsistencias en los diagnósticos de la condición de la mucosa gástrica al momento de la erradicación. Una RS que incluyó artículos primarios que aportaron casi 7000 sujetos, concluyó en que el tratamiento de erradicación de HP en pacientes con etapas primarias en la cascada de Correa como gastritis crónica y AG tendrían disminución del riesgo de CG; lo que ocurre en pacientes con MI y DIS; con un RR de 0,90 (IC95\% 0,43; 1,87) (Chen et al., 2016). Otras dos RS muestran datos en el mismo sentido, lo que tiende a postular que la presencia de MI sería el punto de no retorno de las LPG para el tratamiento de erradicación de HP (Rokkas et al., 2007; Wang et al., 2011). 
Durante muchos años, lo tradicional en una endoscopía digestiva alta, fue tomar exclusivamente muestras de las lesiones encontradas en la mucosa examinada y en consecuencia recibir un informe histopatológico conteniendo sólo esos resultados. En esa modalidad, muchos pacientes han sido y son subevaluados en su riesgo de ser portadores de LPG; así como en el riesgo potencial de desarrollar CG; porque las LPG son usualmente inaparentes a la visión endoscópica y pasarán desapercibidas al endoscopista si éste no toma muestras para biopsia de la mucosa. En consecuencia, cobra relevancia: 1) un sistema de toma de muestras para biopsias en los sitios que aportan datos de la mucosa gástrica transformada a una LPG; y 2) un sistema que pueda expresar el grado de transformación a LPG en extensión e intensidad de mucosa afectada idealmente expresado en etapas. Además, se debe señalar que el cambio de enfoque fundamental es de los endoscopistas, pues son quienes deben tomar la decisión de caracterizar el estómago de los sujetos en riesgo de desarrollar LPG.

De este modo en respuesta al punto $\mathrm{N}^{\circ} 1$, se cuenta con el sistema Sidney modificado y en respuesta al №2 los sistemas: OLGA-OLGIM. A fin de poner a disposición de los clínicos una perfecta comprensión de la evaluación de las LPG. A continuación, se revisarán los tres instrumentos señalados, que son los utilizados para graduar el riesgo individual, y que representan el insumo para la caracterización morfológica de las LPG.

Sistema Sidney modificado para toma de biopsias en el estómago. Se han publicado diversas ubicaciones anatómicas en el estómago, para mapear la mucosa gástrica de forma sistemática y representativa. Ellos, consideran la idea general que tanto la mucosa oxíntica como la antral deben ser "explorados", sumando a ello la mucosa de la incisura angular, considerada altamente expresiva en cuanto mostrar la transformación atrófica-metaplásica precozmente (Rugge et al.). El sistema Sidney que vio la luz en en el Congreso Mundial de Gastroenterología en la ciudad del mismo nombre en 1990, tenía el objetivo final de generar un lenguaje común que plasmara los nuevos conocimientos sobre la biología y el curso natural de gastritis crónica, de especial interés en la época tras el descubrimiento del rol patológico del HP en la gastritis. También perseguía dar pautas sencillas para la documentación de los aspectos microscópicos en muestras de biopsia junto con una clasificación fácilmente comprensible para fines clínicos y de investigación (Misiewicz, 1991; Tytgat, 1991; Sipponen \& Price, 2011). Posteriormente, se publicó el "Sistema Sidney actualizado" (Dixon et al., 1996; Sipponen \& Price), que es un documento que comunica el consenso de Houston y que agrega la necesidad de tomar muestras para biopsia en el ángulo gástrico (incisura angular), e introduce el sistema de escalas analógicas visuales que consisten en un conjunto de dibujos que representan las cinco características de la gastritis que se debe evaluar para determinar densidad de HP, intensidad de la infiltración de células polimorfonucleares y mononucleares, extensión de MI, y gravedad de la AG. Cada panel representa la progresiva intensidad de una característica, desde su ausencia (mucosa normal) hasta el grado intenso o grave. Este protocolo actualizado es el que se usa con más frecuencia por los distintos grupos de trabajo en patología gástrica.

OLGA (Operative Link on Gastritis Assessment). En 2008 un grupo de patólogos, propuso utizar un sistema para informar la AG en etapas que van de 0 a IV. Dichas etapas, dan cuenta de la intensidad de AG y la extensión de la misma constituyendo fenotipos histológicos de $\mathrm{AG}$ en una escala en la que el riesgo de desarrollo de CG va ascendiendo, desde el más bajo (etapa 0) hasta el más alto (etapa IV) (Rugge et al.). La propuesta OLGA (consistente con el protocolo actualizado del sitema Sidney en Houston), recomienda la toma de al menos 5 muestras de biopsia (Fig. 5): dos en el antro pilórico (A1-A2= mucosa secretora) y cuerpo gástrico proximal $(\mathrm{C} 1-\mathrm{C} 2=$ mucosa oxíntica, una anterior y otra posterior); y una en el ángulo (incisura angular), que se considera muy informativo ( $\mathrm{A} 3=$ curvatura menor en la incisura angular, que es el lugar en el que ocurren los cambios atrofia-metaplasia con mayor frecuencia) (Rugge et al.; Rollán et al.). Esta propuesta persigue establecer el inicio de la transformación atrófica-metaplásica de forma precoz. Detalles prácticos de su aplicación, son que las muestras de antro pilórico se toman a 3-4 $\mathrm{cm}$ de píloro, una en la curvatura menor y la otra en la curvatura mayor. La muestra angular no merece ser detallada, en tanto las del cuerpo gástrico, se deben tomar en cara anterior y posterior, o como reza el protocolo más actualizado en curvatura mayor zona media y en curvatura menor a $4 \mathrm{~cm}$ cefálico al ángulo (incisura angular). Existen variaciones de éste, como tomar un mayor número de muestras para mejor caracterización del órgano; lo que trae consigo mayor información, pero también mayor requerimiento de tiempos y costes. Las muestras se deben enviar individualizadas, al menos en 3 cubículos diferentes; esto aún cuando para definir la etapa OLGA, sólo se requiera mucosa antral y corporal separadas; ello porque la mucosa angular se considera dentro de la mucosa antral. Con estos datos se puede definir una etapa para cada paciente con AG y de acuerdo a ello planificar un seguimiento (Fig. 6).

\section{OLGIM (Operative Link on Gastric Intestinal} Metaplasia). De acuerdo a la creciente tendencia de ir identificando pacientes con grados diferentes de riesgo de desarrollo de CG, y así planificar la vigilancia de unos y otros en cuanto a prevención secundaria, es necesario contar con un sistema de gradación de riesgo estándar (inicialmente OLGA, 


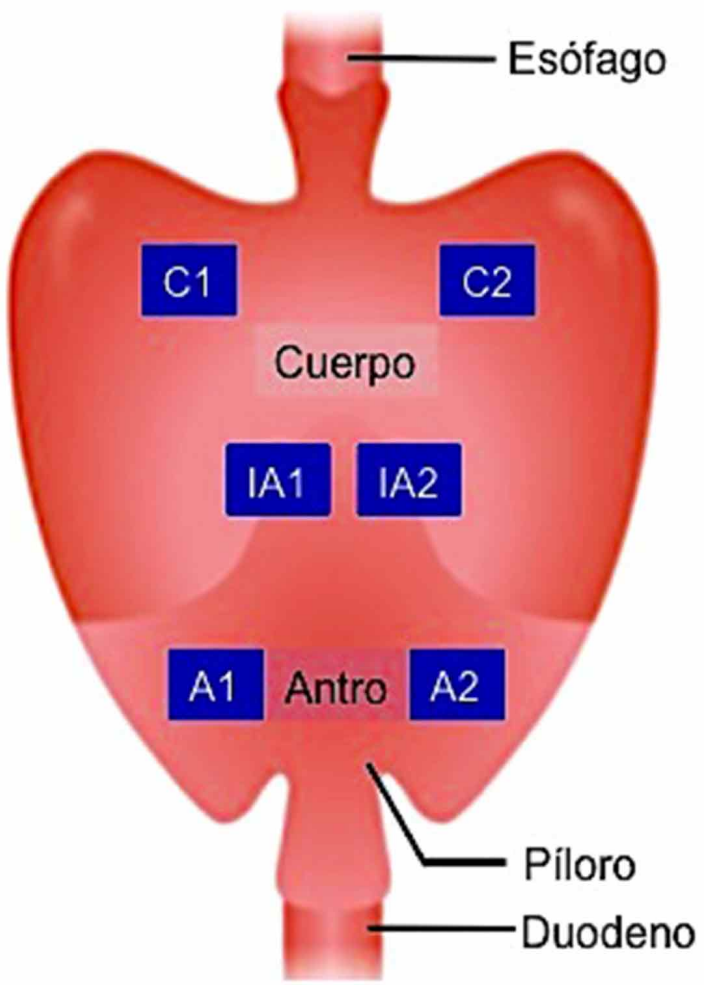

Fig. 5. La propuesta OLGA, recomienda toma de biopsias en el antro pilórico (A1-A2), cuerpo gástrico proximal (C1-C2); y en la incisura angular (IA1).

pero que presenta bajo acuerdo interobservador, incluso tras la inclusión de escalas analógicas visuales para su uso, como se definió en Houston) (el-Zimaity et al., 1996; Offerhaus et al., 1999). Por su parte, la MI se caracteriza por un patrón de células presentes en intestino y fácilmente diferenciable de la histología gástrica, con la consecuencia de un alto grado de acuerdo interobservador, para un sistema que utilice MI y no AG (Guarner et al., 1999; Capelle et al., 2010). Sin embargo, una RS publicada recientemente concluye que existe una asociación significativa entre las etapas III y IV de OLGA y OLGIM, y el riesgo de CG (Yue et al., 2018). Desde el punto de vista de requerimientos técnicos para OLGIM serían menores que para OLGA.
Factores de riesgo para LPG: El paso previo para prevenir el desarrollo de CG, es prevenir la aparición de LPG. Al respecto, existe evidencia referente a que edad, tabaquismo, obesidad, consumo de alcohol, infección por HP y reflujo biliar son factores de riesgo para el desarrollo de MI (de Vries et al.). Además, se han reportado otros factores de riesgo para el desarrollo de LPG: edad avanzada (OR: 2,35; IC95\% 2,07; 2,66); sexo masculino (OR: 1,39; IC95\% 1,29; 1,50); obesidad (OR: 1,10; IC95\% 1,01; 1,18); diabetes mellitus (OR: 1,20; IC95\% 1,00; 1,43); úlcera gástrica (OR: 1,79); la reflujo biliar (OR: 1,06); infección por HP (OR: 1,40; IC95\% 1,29; 1,50); dieta salada y picante (OR: 1,12; IC95\% 1,03; 1,20); AG (OR: 108,69); y DIS (OR: 3,9). También se identificó al producto bruto per cápita como factor de riesgo independiente (den Hoed et al., 2011; Felley et al., 2012; Park \& Kim, 2015; Jiang et al., 2016). Sin embargo, se ha constatado que algunos factores de riesgo para el desarrollo de LPG, varían de acuerdo a la edad y sexo; de tal forma que, en menores de 40 años, el HP sería el único factor de riesgo presente (OR: 3,65; IC95\% 0,42; 31,67); y en mayores de 40 años, sería la diabetes mellitus (OR: 1,23; IC95\% 1,04; 1,45) y la dieta salada y picante (OR: 1,$14 ;$ IC95\% 1,05; 1,22). Por otra parte, en mujeres aparece la obesidad como factor de riesgo independiente (OR: 1,44; IC95\% 1,23; 1,67) (Park et al., 2016).

Por otra parte, existe evidencia que sostiene que ser familiar de primer grado de paciente con CG resulta en una mayor prevalencia de AG (34,0\%) que en sujetos controles con dispepsia no ulcerosa $(5,0 \%)$ a igual prevalencia de HP (El-Omar et al., 2000). Una RS concluyó que familiares de primer grado de pacientes con CG tenían mayor riesgo de tener AG e IM (OR de 2,2 y 1,98 respectivamente) (Rokkas et al. 2010).

Respecto de la DIS, un estudio permitió verificar asociación entre DIS e la historia paterna de CG (OR de 1,88; IC95\% 1,20; 2,95) (You et al., 2000). Además, existe evidencia dada por un estudio de casos y controles, de aso-

\begin{tabular}{|c|l|c|c|c|c|}
\cline { 3 - 6 } \multicolumn{2}{c|}{} & \multicolumn{4}{c|}{ CUERPO GASTRICO } \\
\hline \multirow{3}{*}{ A } & Graduación de la atrofia & Sin atrofia & Atrofia leve & Atrofia moderada & Atrofia intensa \\
\cline { 2 - 6 } $\mathbf{N}$ & Sin atrofia & Estadio O & Estadio I & Estadio II & Estadio II \\
\cline { 2 - 6 } $\mathbf{T}$ & Atrofia leve & Estadio I & Estadio I & Estadio II & Estadio III \\
$\mathbf{R}$ & Atrofia moderada & Estadio II & Estadio II & Estadio III & Estadio IV \\
$\mathbf{O}$ & Astrofia intensa & Estadio III & Estadio III & Estadio IV & Estadio IV \\
\cline { 2 - 6 } & Atro & & & &
\end{tabular}

Fig. 6. Relación de la graduación de la AG entre el antro y cuerpo gástrico, y la gravedad medida en estadios 0 al IV. 
ciación entre infección por HP, OLGA/ OLGIM de alto riesgo y existencia de DIS; con ser familiar de grado de paciente con CG. En este estudio, se diagnosticó además AG en el 70,0 \% de los casos y sólo en el 32,0 \% de los controles ( $\mathrm{p}<0,001)$; infección por HP en el 82,0 \% de los casos vs. $65,0 \%$ de los controles ( $\mathrm{p}=0,001)$; y DIS en 7 pacientes infectados con HP vs. 0 en los controles $(\mathrm{p}=0,007)$ (Marcos-Pinto et al., 2012).

\section{DISCUSIÓN}

En una revisión de estudios poblacionales de Oriente, se constató que la radiografía de doble contraste con bario tiene una baja sensibilidad y alta especificidad, con una tasa de pesquiza inferior en programas de detección masivo, al compararla con la endoscopía realizada a intervalos de menos de dos años. En este sentido, existe evidencia respecto que si los intervalos son mayores a dos años con cualquiera de los dos métodos, sólo aumenta la pesquiza de CG avanzado (Khanderia et al., 2016).

Lo anterior y la evidencia histórica, permiten avalar el hecho que los hallazgos endoscópicos y la confirmación histológica siguen siendo el estándar de referencia para la detección y diagnóstico precoz del CG (Hosokawa et al., 2001; Triantafillidis \& Cheracakis, 2004; Pasechnikov et al., 2005; Rugge, 2007; Rollán et al.; Uedo \& Yao, 2016; Sumiyama, 2017).

En un estudio que buscaba evaluar el efecto del tamizaje con endoscopía en población coreana sobre el resultado de los tratamientos y sus modalidades en CG, concluyó que aumentó al doble la proporción de resecciones locales mediante disección submucosa y en 5 veces los tratamientos laparoscópicos, lo que se tradujo en incremento de supervivencia global a 5 años y mejor calidad de vida postoperatoria (Kim et al., 2014). En el mismo sentido, se ha verificado que la supervivencia a 5 años decrece notablemente si el diagnóstico no es en etapas precoces, lo que refuerza el concepto de la relevancia de la detección temprana en población asintomática (Rollán et al.; Venerito \& Malfertheiner, 2015; Kim et al., 2016; Saumoy et al., 2018). En este mismo sentido, existe evidencia que permite sostener que es posible diagnosticar hasta un $19 \%$ de pacientes con CG en una endoscopía realizada tres años después de una informada como normal (Hosokawa et al., 1998).

En relación a LPG y riesgo de desarrollar un CG, existe evidencia que de los sujetos a los que se les realiza endoscopía con biopsias por indicación clínica, 1 de cada
256 pacientes con mucosa normal, 1 de cada 85 con gastritis, 1 de cada 50 con GA, 1 de cada 39 con MI y 1 de cada 19 con DIS; desarrollarán un CG en los siguientes 20 años (Song et al., 2015).

El cuerpo gástrico de la evidencia presentado en esta revisión, permite afirmar que es posible seleccionar por medio de estudios endoscópicos y biopsias planificadas, individuos con riesgos diferentes de desarrollar CG, estratificando estas poblaciones para eventuales campañas de tamizaje.

Un estudio reciente demostró que la probabilidad de progresión a CG de estadio OLGIM alto (III y IV) vs. bajo (I y II), es dos veces mayor que el de las etapas OLGA altas (III y IV) vs. bajas (I y II) (Mera et al., 2018).

En un estudio de casos y controles se verificó que la AG y la MI localizadas en la mucosa del antro pilórico y cuerpo gástrico, constituyen etapas avanzadas en la estratificación de riesgo individual para el desarrollo de CG, lo que es expresado en los estadios OLGA III/IV en caso AG y OLGIM III/IV en el caso de MI (Zhou et al., 2016); no obstante lo cual, existe evidencia que orienta a que la MI aún en forma aislada (OLGIM I), se asocia a riesgo de CG (OLGA III: OR de 2,09; OLGA IV: OR de 2,04; OLGIM I: OR de 2,38, OLGIM II: OR de 2,97; OLGIM III: OR de 7;89; OLGIM IV: OR de 13,20), destacando el hecho que el OR de OLGIM I es mayor que el de OLGA III (Cho et al., 2013b).

Por otro lado se debe recordar que en cualquier recomendación de seguimiento o de vigilancia endoscópica, se ha de considerar la prescencia de MII como una condición de mayor riesgo de desarrollo de CG que la MIC; así como el antecedente de ser familiar de primer grado de un paciente que ha padecido CG.

De lo anterior se desprende que en países de alto riesgo de CG con recursos limitados, al momento de invertir en estudios de pacientes de riesgo para CG, una endoscopía digestiva alta que solo descarte presencia de CG precoz y más aún sólo CG avanzado será insuficiente y por que no decir estéril en términos de construir grupos de riesgo a quienes aplicar un tamizaje palnificado, regional o nacional. Por todo lo anteriormente expuesto, es que resulta fundamental comunicar y desarrollar las herramientas de estratificación de riesgo de CG que como núcleo concentran la comprensión y tratamiento de las LPG. Es así como en una RS reciente realizada a partir de estudios asiáticos, se concluyó que la detección endoscópica puede reducir el riesgo de muerte por $\mathrm{CG}$, sin afectar la incidencia (Zhang et al., 2018). 
SOTELO, S. \& MANTEROLA, C. Morphology and diagnostic determinants of gastric precancerous conditions. Int. J. Morphol., 37(3)917-927, 2019.

SUMMARY: Gastric carcinoma (GC) of intestinal type, originates from a dysplastic epithelium, which in turn develops in the midst of gastric atrophy (GA) and intestinal metaplasia (IM). Helicobacter pylori (HP) infection is the most frequent cause of GA, causing a multifocal atrophic pangastritis. Among other conditions that produce chronic inflammation of gastric mucosa are also autoimmune gastritis and pernicious anemia. The conceptual framework on which much of current research rests and our understanding of the changes that occur in the gastric mucosa is due to the so-called "Correa waterfall"; who stated that gastric mucosa chronically inflamed, gives way to the GA, which is acquiring foci of IM and in said epithelium a dysplasia (DIS) will eventually develop. The term precancerous conditions (PCC) of the gastric mucosa have been coined to refer to: GA, IM and DIS. After HP eradication, a general reduction in the incidence of GC has been demonstrated; effect that is not so clear, when pangastritis by HP has evolved to extensive GA. Thus, the effect of HP eradication measured through clinical trials has been inconsistent. Severe GA diagnosed represents the highest risk condition. On the other hand, IM can be enteric (grade I), enterocolic (grade II) or colonic (grade III); considering IM III as the variety with the worst prognosis. Histological diagnosis of gastric PCC, determines that the one who suffers them, must undergo endoscopic surveillance. The aim of this manuscript was to update morphological aspects and diagnostic-therapeutic scope of gastric PCC.

KEY WORD: "Precancerous Conditions"[Mesh]; "Stomach Neoplasms"'[Mesh]; "Helicobacter pylori"[Mesh]; Gastric precancerous conditions; "Gastritis, Atrophic"[Mesh]; Gastritis chronic active non atrophic; Gastritis atrophic multifocal; "Metaplasia"[Mesh]; Intestinal metaplasia; Gastric dysplasia.

\section{REFERENCIAS BIBLIOGRÁFICAS}

Abangah, G.; Rahmani, A.; Hafezi-Ahmadi, M. R.; Emami, T.; Asadollahi, K.; Jaafari-Haidarlo, A. \& Moradkhani, A. Precancerous histopathologic lesions of upper gastrointestinal tract among dyspeptic patients upon endoscopic evaluations. J. Gastrointest. Cancer, 47(1):1-7, 2016.

Al-Awadhi, H.; John, R.; Al-Marzooqi, F.; Vincze, A.; Branicki, F. \& Karam, S. M. Sequential alterations in gastric biopsies and tumor tissues support the multistep process of carcinogenesis. Histol. Histopathol., 26(9):1153-64, 2011.

Capelle, L. G.; de Vries, A. C.; Haringsma, J.; Ter Borg, F.; de Vries, R. A.; Bruno, M. J.; van Dekken, H.; Meijer, J.; van Grieken, N. C. \& Kuipers, E. J. The staging of gastritis with the OLGA system by using intestinal metaplasia as an accurate alternative for atrophic gastritis. Gastrointest. Endosc., 71(7):1150-8, 2010.

Chen, H. N.; Wang, Z.; Li, X. \& Zhou, Z. G. Helicobacter pylori eradication cannot reduce the risk of gastric cancer in patients with intestinal metaplasia and dysplasia: evidence from a meta-analysis. Gastric Cancer, 19(1):166-75, 2016.

Cho, S. J.; Choi, I. J.; Kook, M. C.; Nam, B. H.; Kim, C. G.; Lee, J. Y.; Ryu, K. W. \& Kim, Y. W. Staging of intestinal- and diffuse-type gastric cancers with the OLGA and OLGIM staging systems. Aliment. Pharmacol. Ther., 38(10):1292-302, 2013a.

Cho, S. J.; Choi, I. J.; Kook, M. C.; Yoon, H.; Park, S.; Kim, C. G.; Lee, J. Y.; Lee, J. H.; Ryu, K. W. \& Kim, Y. W. Randomised clinical trial: the effects of Helicobacter pylori eradication on glandular atrophy and intestinal metaplasia after subtotal gastrectomy for gastric cancer. Aliment. Pharmacol. Ther, 38(5):477-89, 2013 b.

Correa, P. \& Piazuelo, M. B. The gastric precancerous cascade. J. Dig. Dis., 13(1):2-9, 2012.

Correa, P. A human model of gastric carcinogenesis. Cancer Res. 48(13):3554-60, 1998.

Correa, P. Human gastric carcinogenesis: a multistep and multifactorial process--First American Cancer Society Award Lecture on Cancer Epidemiology and Prevention. Cancer Res., 52(24):6735-40, 1992.

Correa, P.; Haenszel, W.; Cuello, C.; Tannenbaum, S. \& Archer, M. A model for gastric cancer epidemiology. Lancet., 2(7924):58-60, 1975.

de Vries, A. C.; van Grieken, N. C.; Looman, C. W.; Casparie, M. K.; de Vries, E.; Meijer, G. A. \& Kuipers, E. J. Gastric cancer risk in patients with premalignant gastric lesions: a nationwide cohort study in the Netherlands. Gastroenterology, 134(4):945-52, 2008.

den Hoed, C. M.; Holster, I. L.; Capelle, L. G.; de Vries, A. C.; den Hartog, B.; Ter Borg, F.; Biermann, K. \& Kuipers, E. J. Follow-up of premalignant lesions in patients at risk for progression to gastric cancer. Endoscopy, 45(4):249-56, 2013.

den Hoed, C. M.; van Eijck, B. C.; Capelle, L. G.; van Dekken, H.; Biermann, K.; Siersema, P. D. \& Kuipers, E. J. The prevalence of premalignant gastric lesions in asymptomatic patients: predicting the future incidence of gastric cancer. Eur. J. Cancer, 47(8):1211-8, 2011.

Dinis-Ribeiro, M.; Areia, M.; de Vries, A.C.; Marcos-Pinto, R.; MonteiroSoares, M.; O'Connor, A.; Pereira, C.; Pimentel-Nunes, P.; Correia, R.; Ensari, A.; et al. Management of precancerous conditions and lesions in the stomach (MAPS): guideline from the European Society of Gastrointestinal Endoscopy (ESGE), European Helicobacter Study Group (EHSG), European Society of Pathology (ESP), and the Sociedade Portuguesa de Endoscopia Digestiva (SPED). Endoscopy, 44(1):74-94, 2012.

Dixon, M. F.; Genta, R. M.; Yardley, J. H. \& Correa, P. Classification and grading of gastritis. The updated Sydney System. International Workshop on the Histopathology of Gastritis, Houston 1994. Am. J. Surg. Pathol., 20(10):1161-81, 1996.

El-Omar, E. M.; Oien, K.; Murray, L. S.; El-Nujumi, A.; Wirz, A.; Gillen, D.; Williams, C.; Fullarton, G. \& McColl, K. E. Increased prevalence of precancerous changes in relatives of gastric cancer patients: critical role of H. pylori. Gastroenterology, 118(1):22-30, 2000.

el-Zimaity, H. M.; Graham, D. Y.; al-Assi, M. T.; Malaty, H.; Karttunen, T. J.; Graham, D. P.; Huberman, R. M. \& Genta, R. M. Interobserver variation in the histopathological assessment of Helicobacter pylori gastritis. Hum. Pathol., 27(1):35-41, 1996.

Felley, C.; Bouzourene, H.; VanMelle, M. B.; Hadengue, A.; Michetti, P.; Dorta, G.; Spahr, L.; Giostra, E. \& Frossard, J. L. Age, smoking and overweight contribute to the development of intestinal metaplasia of the cardia. World J. Gastroenterol., 18(17):2076-83, 2012.

Fukase, K.; Kato, M.; Kikuchi, S.; Inoue, K.; Uemura, N.; Okamoto, S.; Terao, S.; Amagai, K.; Hayashi, S.; Asaka, M.; et al. Effect of eradication of Helicobacter pylori on incidence of metachronous gastric carcinoma after endoscopic resection of early gastric cancer: an open-label, randomised controlled trial. Lancet, 372(9636):392-7, 2008.

González, C. A.; Sanz-Anquela, J. M.; Companioni, O.; Bonet, C.; Berdasco, M.; López, C.; Mendoza, J.; Martín-Arranz, M. D.; Rey, E.; Poves, E.; et al. Incomplete type of intestinal metaplasia has the highest risk to progress to gastric cancer: results of the Spanish follow-up multicenter study. J. Gastroenterol. Hepatol., 31(5):953-8, 2016.

González, C. A.; Sanz-Anquela, J. M.; Gisbert, J. P. \& Correa, P. Utility of subtyping intestinal metaplasia as marker of gastric cancer risk. A review of the evidence. Int. J. Cancer, 133(5):1023-32, 2013. 
Guarner, J.; Herrera-Goepfert, R.; Mohar, A.; Sanchez, L.; Halperin, D.; Ley, C. \& Parsonnet, J. Interobserver variability in application of the revised Sydney classification for gastritis. Hum. Pathol., 30(12):14314, 1999.

Hosokawa, O.; Tsuda, S.; Kidani, E.; Watanabe, K.; Tanigawa, Y.; Shirasaki, S.; Hayashi, H. \& Hinoshita, T. Diagnosis of gastric cancer up to three years after negative upper gastrointestinal endoscopy. Endoscopy, 30(8):669-74, 1998.

Hosokawa, O.; Watanabe, K.; Hatorri, M.; Douden, K.; Hayashi, H. \& Kaizaki, Y. Detection of gastric cancer by repeat endoscopy within a short time after negative examination. Endoscopy, 33(4):301-5, 2001.

Jiang, J. X.; Liu, Q.; Zhao, B.; Zhang, H. H.; Sang, H. M.; Djaleel, S. M.; Zhang, G. X. \& Xu, S. F. Risk factors for intestinal metaplasia in a southeastern Chinese population: an analysis of 28,745 cases. J. Cancer Res. Clin. Oncol., 143(3):409-18, 2016.

Khanderia, E.; Markar, S. R.; Acharya, A.; Kim, Y.; Kim, Y. W. \& Hanna, G. B. The influence of gastric cancer screening on the stage at diagnosis and survival: a meta-analysis of comparative studies in the Far East. J. Clin. Gastroenterol., 50(3):190-7, 2016.

Kim, G. H.; Liang, P. S.; Bang, S. J. \& Hwang, J. H. Screening and surveillance for gastric cancer in the United States: Is it needed? Gastrointest. Endosc., 84(1):18-28, 2016.

Kim, Y. G.; Kong, S. H.; Oh, S. Y.; Lee, K. G.; Suh, Y. S.; Yang, J. Y.; Choi, J.; Kim, S. G.; Kim, J. S.; Kim, W. H.; et al. Effects of screening on gastric cancer management: comparative analysis of the results in 2006 and in 2011. J. Gastric Cancer, 14(2):129-34, 2014.

Kuipers, E. J. Review article: Relationship between Helicobacter pylori, atrophic gastritis and gastric cancer. Aliment. Pharmacol. Ther, 12 Suppl. 1:25-36, 1998.

Kuipers, E. J.; Uyterlinde, A. M.; Peña, A. S.; Roosendaal, R.; Pals, G.; Nelis, G. F.; Festen, H. P. \& Meuwissen, S. G. Long-term sequelae of Helicobacter pylori gastritis. Lancet, 345(8964):1525-8, 1995.

Lage, J.; Uedo, N.; Dinis-Ribeiro, M. \& Yao, K. Surveillance of patients with gastric precancerous conditions. Best Pract. Res. Clin. Gastroenterol., 30(6):913-22, 2016.

Lahner, E.; Esposito, G.; Pilozzi, E.; Purchiaroni, F.; Corleto, V. D.; Di Giulio, E. \& Annibale, B. Occurrence of gastric cancer and carcinoids in atrophic gastritis during prospective long-term follow up. Scand. J. Gastroenterol., 50(7):856-65, 2015.

Li, D.; Bautista, M. C.; Jiang, S. F.; Daryani, P.; Brackett, M.; Armstrong, M. A.; Hung, Y. Y.; Postlethwaite, D. \& Ladabaum, U. Risks and predictors of gastric adenocarcinoma in patients with gastric intestinal metaplasia and dysplasia: a population-based study. Am. J. Gastroenterol., 111(8):1104-13, 2016.

Lim, H.; Jung, H. Y.; Park, Y. S.; Na, H. K.; Ahn, J. Y.; Choi, J. Y.; Lee, J. H.; Kim, M. Y.; Choi, K. S.; Kim, D. H.; et al. Discrepancy between endoscopic forceps biopsy and endoscopic resection in gastric epithelial neoplasia. Surg. Endosc., 28(4):1256-62, 2014.

Marcos-Pinto, R.; Carneiro, F.; Dinis-Ribeiro, M.; Wen, X.; Lopes, C.; Figueiredo, C.; Machado, J. C.; Ferreira, R. M.; Reis, C. A.; Ferreira, J.; et al. First-degree relatives of patients with early-onset gastric carcinoma show even at young ages a high prevalence of advanced OLGA/OLGIM stages and dysplasia. Aliment. Pharmacol. Ther, 35(12):1451-9, 2012.

Marques-Silva, L.; Areia, M.; Elvas, L. \& Dinis-Ribeiro, M. Prevalence of gastric precancerous conditions: a systematic review and meta-analysis. Eur. J. Gastroenterol. Hepatol., 26(4):378-87, 2014.

Mera, R. M.; Bravo, L. E.; Camargo, M. C.; Bravo, J. C.; Delgado, A. G.; Romero-Gallo, J.; Yepez, M. C.; Realpe, J. L.; Schneider, B. G.; Morgan, D. R.; et al. Dynamics of Helicobacter pylori infection as a determinant of progression of gastric precancerous lesions: 16-year follow-up of an eradication trial. Gut, 67(7):1239-46, 2018.

Misiewicz, J. J. The Sydney System: a new classification of gastritis. Introduction. J. Gastroenterol. Hepatol., 6(3):207-8, 1991.

Offerhaus, G. J.; Price, A. B.; Haot, J.; ten Kate, F. J.; Sipponen, P.; Fiocca, R.; Stolte, M. \& Dixon, M. F. Observer agreement on the grading of gastric atrophy. Histopathology, 34(4):320-5, 1999.
Park, Y. H. \& Kim, N. Review of atrophic gastritis and intestinal metaplasia as a premalignant lesion of gastric cancer. J. Cancer Prev., 20(1):2540, 2015.

Park, Y. M.; Kim, J. H.; Baik, S. J.; Park, J. J.; Youn, Y. H. \& Park, H. Clinical risk assessment for gastric cancer in asymptomatic population after a health check-up: An individualized consideration of the risk factors. Medicine (Baltimore), 95(44):e5351, 2016.

Pasechnikov, V. D.; Chukov, S. Z.; Kotelevets, S. M.; Mostovov, A. N.; Mernova, V. P. \& Polyakova, M. B. Invasive and non-invasive diagnosis of Helicobacter pylori-associated atrophic gastritis: a comparative study. Gastroenterology, 40(3):297-301, 2005.

Pimentel-Nunes, P.; Libânio, D.; Marcos-Pinto, R.; Areia, M.; Leja, M.; Esposito, G.; Garrido, M.; Kikuste, I.; Megraud, F.; Matysiak-Budnik, T.; et al. Management of epithelial precancerous conditions and lesions in the stomach (MAPS II): European Society of Gastrointestinal Endoscopy (ESGE), European Helicobacter and Microbiota Study Group (EHMSG), European Society of Pathology (ESP), and Sociedade Portuguesa de Endoscopia Digestiva (SPED) guideline update 2019. Endoscopy, 51(4):365-88, 2019.

Rokkas, T.; Pistiolas, D.; Sechopoulos, P.; Robotis, I. \& Margantinis, G. The long-term impact of Helicobacter pylori eradication on gastric histology: a systematic review and meta-analysis. Helicobacter, 12 Suppl. 2:32-8, 2007.

Rokkas, T.; Sechopoulos, P.; Pistiolas, D.; Margantinis, G. \& Koukoulis, G. Helicobacter pylori infection and gastric histology in first-degree relatives of gastric cancer patients: a meta-analysis. Eur. J. Gastroenterol. Hepatol., 22(9):1128-33, 2010.

Rollán, A.; Cortés, P.; Calvo, A.; Araya, R.; Bufadel, M. E.; González, R.; Heredia, C.; Muñoz, P.; Squella, F.; Nazal, R.; et al. Recommendations of the Chilean Association for Digestive Endoscopy for the management of gastric pre-malignant lesions. Rev. Med. Chil., 142(9):1181-92, 2014.

Rugge, M. Secondary prevention of gastric cancer. Gut, 56(12):1646-7, 2007.

Rugge, M.; Correa, P.; Di Mario, F.; El-Omar, E.; Fiocca, R.; Geboes, K.; Genta, R. M.; Graham, D. Y.; Hattori, T.; Malfertheiner, P.; et al. OLGA staging for gastritis: a tutorial. Dig. Liver Dis., 40(8):650-8, 2008.

Sampieri, C. L. Helicobacter pylori and gastritis: the role of extracellular matrix metalloproteases, their inhibitors, and the disintegrins and metalloproteases--a systematic literature review. Dig. Dis. Sci., 58(10):2777-83, 2013

Saumoy, M.; Schneider, Y.; Shen, N.; Kahaleh, M.; Sharaiha, R. Z. \& Shah, S. C. Cost Effectiveness of Gastric Cancer Screening According to Race and Ethnicity. Gastroenterology, 155(3):648-60, 2018.

Sipponen, P. \& Price, A. B. The Sydney System for classification of gastritis 20 years ago. J. Gastroenterol. Hepatol., 26 Suppl. 1:31-4, 2011.

Song, H.; Ekheden, I. G.; Zheng, Z.; Ericsson, J.; Nyrén, O. \& Ye, W. Incidence of gastric cancer among patients with gastric precancerous lesions: observational cohort study in a low risk Western population. B. M. J., $351: \mathrm{h} 3867,2015$.

Sugano, K. Premalignant conditions of gastric cancer. J. Gastroenterol. Hepatol., 28(6):906-11, 2013.

Sumiyama, K. Past and current trends in endoscopic diagnosis for early stage gastric cancer in Japan. Gastric Cancer, 20(Suppl. 1):20-7, 2017.

Sung, J. K. Diagnosis and management of gastric dysplasia. Korean J. Intern. Med., 31(2):201-9, 2016.

Take, S.; Mizuno, M.; Ishiki, K.; Nagahara, Y.; Yoshida, T.; Yokota, K. \& Oguma, K. Baseline gastric mucosal atrophy is a risk factor associated with the development of gastric cancer after Helicobacter pylori eradication therapy in patients with peptic ulcer diseases. J. Gastroenterol., 42 Suppl. 17:21-7, 2007

Toh, B. H. Diagnosis and classification of autoimmune gastritis. Autoimmun. Rev., 13(4-5):459-62, 2014.

Triantafillidis, J. K. \& Cheracakis, P. Diagnostic evaluation of patients with early gastric cancer--a literature review. Hepatogastroenterology, 51(56):618-24, 2004.

Tytgat, G. N. The Sydney System: endoscopic division. Endoscopic appearances in gastritis/duodenitis. J. Gastroenterol. Hepatol., 6(3):22334, 1991. 
Uedo, N. \& Yao, K. Endoluminal diagnosis of early gastric cancer and its precursors: bridging the gap between endoscopy and pathology. $A d v$. Exp. Med. Biol., 908:293-316, 2016.

Vannella, L.; Lahner, E.; Osborn, J.; Bordi, C.; Miglione, M.; Delle Fave, G. \& Annibale, B. Risk factors for progression to gastric neoplastic lesions in patients with atrophic gastritis. Aliment. Pharmacol. Ther., 31(9):1042-50, 2010.

Venerito, M. \& Malfertheiner, P. Preneoplastic conditions in the stomach: always a point of no return? Dig. Dis., 33(1):5-10, 2015.

Wang, J.; Xu, L.; Shi, R.; Huang, X.; Li, S. W.; Huang, Z. \& Zhang, G. Gastric atrophy and intestinal metaplasia before and after Helicobacter pylori eradication: a meta-analysis. Digestion, 83(4):253-60, 2011.

Whiting, J. L.; Sigurdsson, A.; Rowlands, D. C.; Hallissey, M. T. \& Fielding, $\mathrm{J}$. W. The long term results of endoscopic surveillance of premalignant gastric lesions. Gut, 50(3):378-81, 2002.

Wolf, E. M.; Plieschnegger, W.; Geppert, M.; Wigginghaus, B.; Höss, G. M.; Eherer, A.; Schneider, N. I.; Hauer, A.; Rehak, P.; Vieth, M.; et al. Changing prevalence patterns in endoscopic and histological diagnosis of gastritis? Data from a cross-sectional Central European multicentre study. Dig. Liver Dis., 46(5):412-8, 2014.

Wong, B. C.; Lam, S. K.; Wong, W. M.; Chen, J. S.; Zheng, T. T.; Feng, R. E.; Lai, K. C.; Hu, W. H.; Yuen, S. T.; Leung, S. Y.; et al. Helicobacter pylori eradication to prevent gastric cancer in a high-risk region of China: a randomized controlled trial. JAMA, 291(2):187-94, 2004.

Yakirevich, E. \& Resnick, M. B. Pathology of gastric cancer and its precursor lesions. Gastroenterol. Clin. North Am., 42(2):261-84, 2013.

Yanaoka, K.; Oka, M.; Ohata, H.; Yoshimura, N.; Deguchi, H.; Mukoubayashi, C.; Enomoto, S.; Inoue, I.; Iguchi, M.; Maekita, T.; et $a l$. Eradication of Helicobacter pylori prevents cancer development in subjects with mild gastric atrophy identified by serum pepsinogen levels. Int. J. Cancer, 125(11):2697-703, 2009.

You, W. C.; Ma, J. L.; Liu, W. D.; Gail, M. H.; Chang, Y. S.; Zhang, L.; Hu, Y. R.; Fraumeni, J. F. Jr. \& Xu, G. W. Blood type and family cancer history in relation to precancerous gastric lesions. Int. J. Epidemiol., 29(3):405-7, 2000.

Yue, H.; Shan, L. \& Bin, L. The significance of OLGA and OLGIM staging systems in the risk assessment of gastric cancer: a systematic review and meta-analysis. Gastric Cancer, 21(4):579-87, 2018.

Zhang, X.; Li, M.; Chen, S.; Hu, J.; Guo, Q.; Liu, R.; Zheng, H.; Jin, Z.; Yuan, Y.; Xi, Y.; et al. Endoscopic screening in Asian countries is associated with reduced gastric cancer mortality: a meta-analysis and systematic review. Gastroenterology, 155(2):347-354.e9, 2018.

Zhou, Y.; Li, H. Y.; Zhang, J. J.; Chen, X. Y.; Ge, Z. Z. \& Li, X. B. Operative link on gastritis assessment stage is an appropriate predictor of early gastric cancer. World J. Gastroenterol., 22(13):3670-8, 2016.

\author{
Dirección para correspondencia: \\ Dr. Carlos Manterola \\ Departamento de Cirugía y CEMyQ \\ Universidad de La Frontera \\ Manuel Montt 112, oficina 408 \\ Temuco \\ CHILE
}

E-mail: carlos.manterola@ufrontera.cl

Recibida : 04-02-2019

Aceptada: 16-04-2019 